\title{
Equivalence principle and quantum statistics
}

\author{
Majumdar A.S. ${ }^{1}$, Mousavi S.V. ${ }^{2}$, Home D. ${ }^{3}$ \\ ${ }^{1}$ S. N. Bose National Center for Basic Sciences, Block JD, Sector III, Salt Lake, Kolkata, India; \\ ${ }^{2}$ Department of Physics, The University of Qom, Qom, Iran; \\ ${ }^{3}$ CAPSS, Department of Physics, Bose Institute, Sector-V, Salt Lake, Kolkata, India; \\ E-mail: Majumdar<archan@bose.res.in>;
}

\begin{abstract}
Violation of the gravitational weak equivalence principle in quantum mechanics (WEQ) has been earlier studied using Gaussian as well as non-Gaussian wave packets in free fall. Here we study the effect of quantum statistics on the arrival time distribution of quantum particles computed through the probability current density. We show that symmetrization or asymmetrization of the wave function impacts the arrival time distribution of wavepackets. The mean arrival time is dependent on the mass of the particles, and varies according to the statistics.
\end{abstract}

Keywords: Weak equivalence principle, Quantum statistics, Arrival time distribution, Spin.

DOI: 10.18698/2309-7604-2015-1-359-364

\section{Introduction}

The motion of freely falling particles is traditionally taken to conform to the weak equivalence principle (WEP) of gravitation which states that all sufficiently small objects fall with the same acceleration independent of their mass and constituent in a uniform gravitational field. WEP is regarded to be a fundamentally classical and local principle. Study of the equivalence principle in quantum mechanics has evoked a lot of interest. A statement of the principle in quantum mechanics is as follows: "The results of experiments in an external potential comprising just a sufficiently weak, homogeneous gravitational field, as determined by the wavefunction, are independent of the mass of the system" [1]. This assertion is also called the weak equivalence principle of quantum mechanics (WEQ).

Various approaches have been used to study the possibility of violation of weak equivalence principle in quantum mechanics, such as the prediction of mass-dependence of the radii, frequencies and binding energy of a particle in an external gravitational field [2]. A gedanken experiment studying the free fall of quantum test particles in a uniform gravitational field predicts mass-dependence of the time of flight distribution [3]. Another approach using a model quantum clock predicts violation of WEQ in the vicinity of the turning point of classical trajectories [4]. Experimental violation has been observed in the interference phenomenon associated with the gravitational potential in neutron and atomic interferometry experiments [5-6]. An explicit mass dependence of the position probabilities has been shown for quantum particles projected upwards against gravity around both the classical turning point and the point of initial projection using 
Gaussian [7] and non-Gaussian [8] wavepackets. Explicit mass dependence of the mean arrival time at an arbitrary detector location has also been predicted for a Gaussian [7] wave-packet under free fall, an effect which may be enhanced using suitably chosen non-Gaussian wavepackets [8].

The violation of WEQ has been established in single particle quantum mechanics. In the present work we are interested to examine the effect of quantum statistics on the WEQ. Several important phenomena based on quantum statistics are experimentally revealed through the measurement of time of flight of quantum particles in free fall [9]. Here we study the effect of statistics on the arrival time distribution of a system of freely falling wavepackets consisting of two identical particles. Consideration of quantum mechanical effects on such time of flight distributions beyond the standard semi-classical analysis could reveal interesting observational effects, as discussed earlier in the literature [10]. Our analysis is based on the probability current approach for computing the mean arrival time distribution of wavepackets [11].

\section{Formalism}

We consider a two-body system composed of two non-interacting identical particles in an external field. Identical particles are classically distinguishable and obey Maxwell-Boltzmann (MB) statistics, while they are indistinguishable in quantum mechanics and obey different statistics. For Fermi-Dirac (Bose-Einstein) statistics the total wavefunction must be antisymmetrized (symmetrized) under the exchange of particles in the system. Since particles do not interact, solutions of the Schrödinger equation are constructed from two single-particle wavefunctions $\psi_{\mathrm{a}}$ and $\psi_{\mathrm{b}}$ as follows [1]

$$
\Psi_{ \pm}\left(\mathrm{z}_{1}, \mathrm{z}_{2}, \mathrm{t}\right)=\mathrm{N}_{ \pm}\left[\Psi_{\mathrm{a}}\left(\mathrm{z}_{1}, \mathrm{t}\right) \Psi_{\mathrm{b}}\left(\mathrm{z}_{2}, \mathrm{t}\right) \pm \Psi_{\mathrm{b}}\left(\mathrm{z}_{1}, \mathrm{t}\right) \Psi_{\mathrm{a}}\left(\mathrm{z}_{2}, \mathrm{t}\right)\right]
$$

where the upper (lower) sign stands for BE (FD) statistics and the normalization constants are given by $N_{ \pm}=\left[2\left(1 \pm\left|\left\langle\Psi_{a}(t) \mid \Psi_{b}(t)\right\rangle\right|^{2}\right)\right]^{-\frac{1}{2}}$.

Here we employ the probability current approach to study the effect of particle statistics on the arrival time distribution of a two-body system. In this approach, the arrival time distribution at a detector location $z=Z$ is given by [11] 


$$
\Pi(\mathrm{Z}, \mathrm{t})=\frac{\left|j_{1}(Z, t)\right|}{\int_{0}^{\infty} d t\left|j_{1}(Z, t)\right|}
$$

As a result one obtains

$$
\tau(Z)=\int_{0}^{\infty} d t t \Pi(\mathrm{Z}, \mathrm{t})
$$

for the mean arrival time at the detector location $z=Z$. Now, using single-particle continuity equation, one obtains the following relation for the one-body probability current density [12]

$$
\begin{aligned}
& j_{1}(z, t)=\frac{\hbar}{m}\left|N_{ \pm}\right|^{2} \Im\left\{\psi_{a}^{*} \frac{\partial \psi_{a}}{\partial z}+\psi_{b}^{*} \frac{\partial \psi_{b}}{\partial z} \pm \psi_{\mathrm{a}}(\mathrm{t}) \mid \psi_{\mathrm{b}}(\mathrm{t}) \psi_{b}^{*} \frac{\partial \psi_{a}}{\partial z} \pm\right. \\
& \left.\psi_{\mathrm{b}}(\mathrm{t}) \mid \psi_{\mathrm{a}}(\mathrm{t}) \psi_{a}^{*} \frac{\partial \psi_{b}}{\partial z}\right\}
\end{aligned}
$$

\section{Arrival time of particles in free fall}

The arrival time of freely falling wavepackets using the probability current approach has been studied earlier [7-8], where it was noted that the arrival time distribution acquires a massdependence due to wavepacket spread. Now we study the effect of symmetrization and asymmetrization of the wavefunction of a system of two particles falling freely under gravity. Here we choose the initial single-particle wavefunctions as Gaussians,

$$
\psi_{i}(z, 0)=\frac{1}{\left(2 \pi \sigma_{0 i}^{2}\right)^{\frac{1}{4}}} \exp \left\{i k_{i} z-\frac{\left(z-z_{c i}\right)^{2}}{4 \sigma_{0 i}^{2}}\right\}, \quad i=a, b
$$


and set the initial velocity of the particles to be zero, i.e., the particles are dropped from rest with $k_{a}=k_{b}=0$, and accelerate downwards under gravity with $g=10 \mathrm{~m} / \mathrm{s}^{2}$. The time-evolved single-particle wavefunctions in the uniform gravitational field $V(z)=m g z$ are given by [13],

$$
\begin{aligned}
& \psi_{i}(z, t)=\frac{1}{\left(2 \pi s_{t i}^{2}\right)^{\frac{1}{4}}} \exp \times \\
& \times\left\{\frac{i m}{2 \hbar t}\left[\left(z^{2}-g t^{2} z-\frac{g^{2} t^{4}}{12}+i \frac{\hbar t}{2 m \sigma_{0 i}^{2}} z_{c i}^{2}\right)-\frac{\sigma_{0 i}}{s_{t i}}\left(z-z_{c i}+\frac{g t^{2}}{2}++\frac{s_{t i}}{\sigma_{0 i}} z_{c i}\right)^{2}\right]\right\}
\end{aligned}
$$

where $s_{t i}=\sigma_{0 i}\left(1+\frac{i \hbar t}{2 m \sigma_{0}^{2}}\right)$. The overlap integral is given by

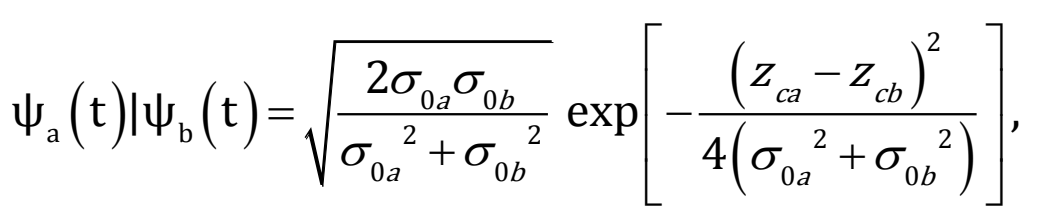

and hence the normalization constants become

$$
\mathrm{N}_{ \pm}=\frac{1}{\sqrt{2}}\left\{1 \pm \frac{2 \sigma_{0 a} \sigma_{0 b}}{\sigma_{0 a}{ }^{2}+\sigma_{0 b}{ }^{2}} \exp \left[-\frac{\left(z_{c a}-z_{c b}\right)^{2}}{4\left({\sigma_{0 a}}^{2}+\sigma_{0 b}{ }^{2}\right)}\right]\right\}^{-\frac{1}{2}}
$$

Using the following values of the parameters $\sigma_{0 a}=\sigma_{0 b}=\sigma_{0}=5 \mu \mathrm{m}, z_{c a}=9 \sigma_{0}, z_{c b}=$ $7 \sigma_{0}, m=m_{n}=1.67 \times 10^{-27} \mathrm{~kg}$ and $t_{r e f}=0.79 \mathrm{~ms}$ for numerical calculations, we have plotted in figure 1 the arrival time distribution at the detector location $Z=0$ by substituting the expression for the time-evolved wavefunction eq.(5) in the expression for the probability current given by eq.(4), and then using eq.(2). As one sees, the particle statistics has an impact on the arrival time distribution. Such an effect of symmetrization and asymmetrization of a two-body wavefunction on arrival times of freely falling wavepackets may be regarded as nonlocal (in the sense that the single-particle arrival time distribution depends on the spatially separated second 
particle, as well), and thus contrary to the tenet of the local weak equivalence principle of classical gravity, which forms the inspiration of the statement of WEQ.

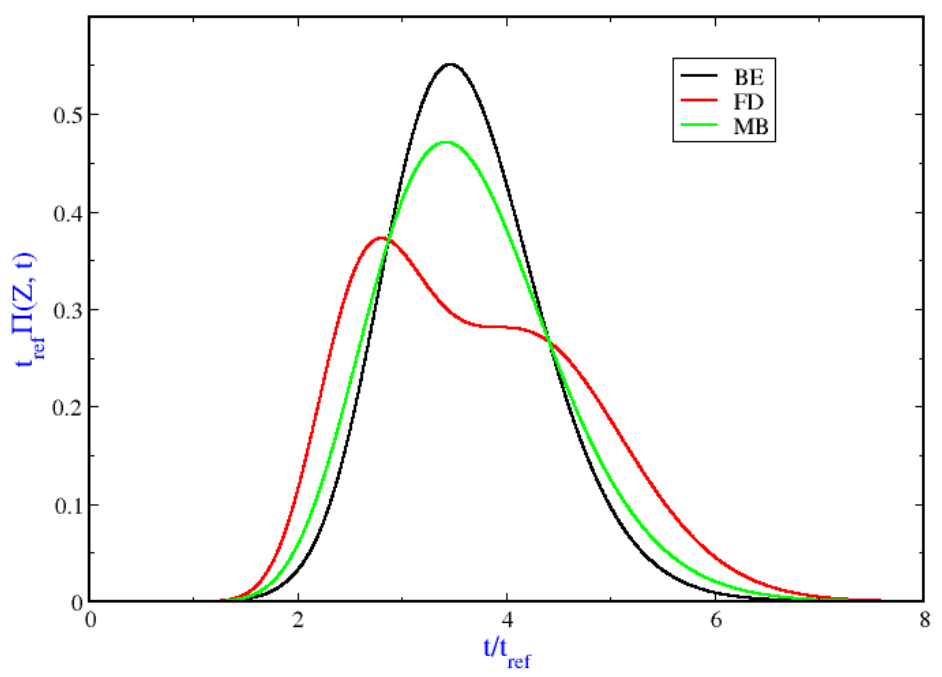

Fig. 1. Arrival time distribution versus time.

The mass dependence of arrival time for particles in free fall is exhibited in the plot of the mean arrival time versus mass in the figure 2 . Here again, the mean arrival time is computed at the detector location $Z=0$ using eq.(2) and eq.(3) after substituting the expression for the timeevolved wavefunction eq.(5) in the expression for the probability current given by eq.(4). The values of the parameters used are as before. One sees that for all types of statistics the mean arrival time decreases with mass at first and then becomes constant for large mass.

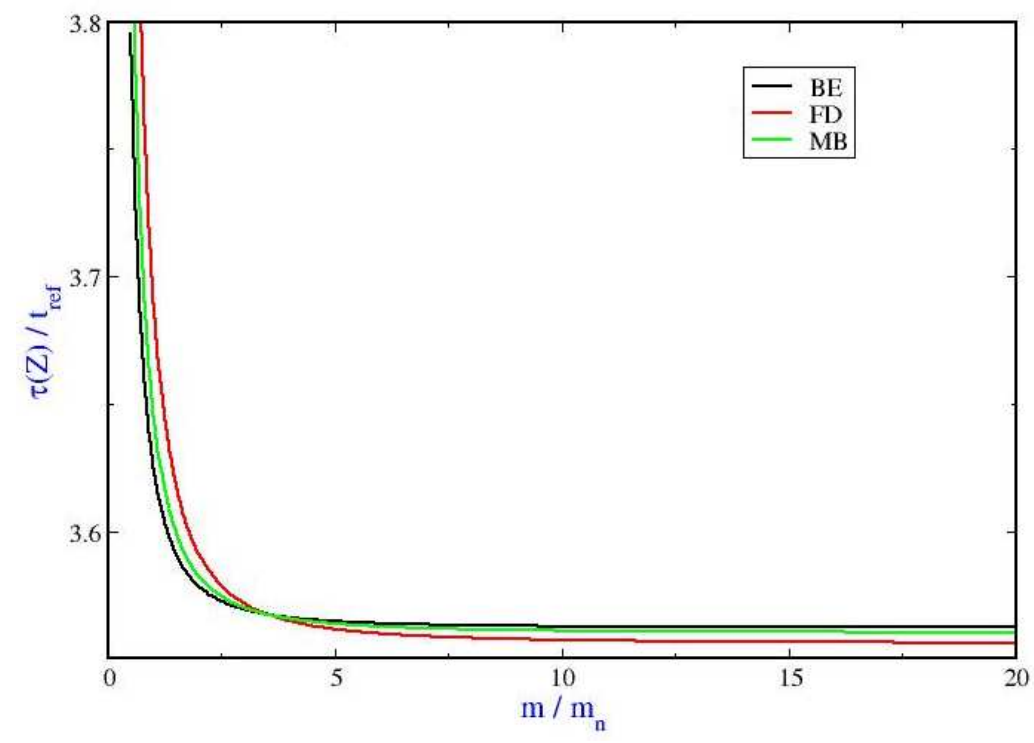

Fig. 2. Mean arrival time versus mass. 


\section{Conclusions}

In this work we have studied some effects of quantum statistics on the equivalence principle in quantum mechanics. Symmetrization and asymmetrization of the wavefunction clearly affects the magnitude of the violation of WEQ. Such an effect is more prominent compared to the effect of violation of WEQ [13] through explicit spin-dependence of the probability current density [14]. However, the violation of WEQ observed explicitly for low masses tends to disappear smoothly in the limit of large mass. The classical limit of the mean arrival time may be computed using the probability current approach [15]. We observe here that even in the presence of particle statistics, the classical limit of mean arrival time emerges smoothly through this approach.

Acknowledgements: ASM and DH acknowledge support from the project SR/S2/LOP-08/2013 of DST, India.

\section{References}

1. Holland P.R. (1993). The Quantum Theory of Motion. Cambridge: Cambridge University

Press. 2. Greenberger D.M., Overhauser A.W. (1979). Rev. Mod. Phys., 51, 43.

3. Viola L., Onofrio R. (1997). Phys. Rev., D 55, 455.

4. Davies P.C.W. (2004). Class. Quantum Grav. 21, 2761.

5. Colella R., Overhauser A.W., Werner S.A. (1975). Phys. Rev. Lett., 34, 1472.

6. Peters A., Chung K.Y., Chu S. (1999). Nature, 400, 849.

7. Ali Md. M., Majumdar A.S., Home D., Pan A.K. (2006). Class. Quant. Grav., 23, 6493

8. Chowdhury P., Home D., Majumdar A.S., Mousavi S.V. (2012). Class. Quant. Grav., 29, 025010 .

9. Anderson M.H. (1995). Science, 269, 198.

10. Ali Md.M., Majumdar A.S., Home D., Pan A.K. (2007). Phys. Rev., A 75, 042110.

11. Dumont R.S., Marchioro T.L. (1993). Phys. Rev., A 47, 85.

12. Mousavi S.V., Miret-Art'es S. (2015). Phys. Scr., 90, 025001.

13. Mousavi S.V., Majumdar A.S., Home D. (2015). arXiv, 1502.07875.

14. Holland P.R. (1999). Phys. Rev., A 60, 4326.

15. Ali Md.M., Majumdar A.S., Pan A.K. (2006). Found. Phys. Lett., 19, 723. 